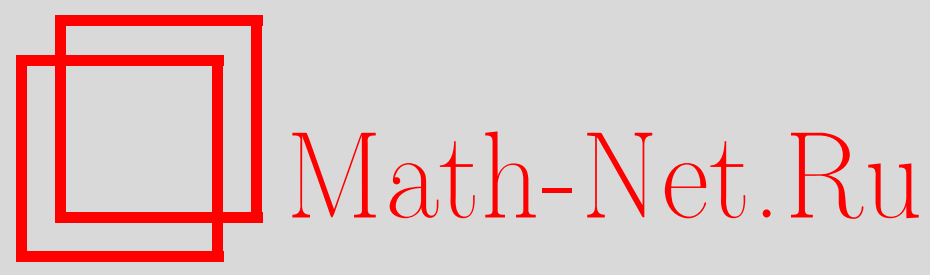

Ю. Э. Линке, Раздельно непрерывные селекторы, Матем. заметки, 1998, том 63, выпуск 2, 209-216

DOI: https://doi.org/10.4213/mzm1267

Использование Общероссийского математического портала Math-Net.Ru подразумевает, что вы прочитали и согласны с пользовательским соглашением http://www.mathnet.ru/rus/agreement

Параметры загрузки:

IP : 52.205.19.152

26 апреля 2023 г., 16:23:00

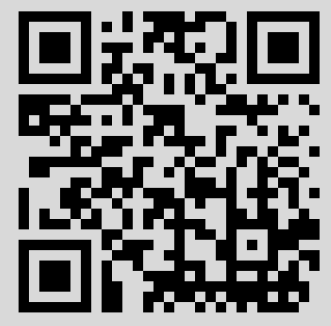


УДК 515.122 .5

\title{
РАЗДЕЛЬНО НЕПРЕРЫВНЫЕ СЕЛЕКТОРЫ
}

\author{
Ю. Э. Линке
}

Доказана теорема о наличии раздельно непрерывных селекторов у раздельно полунепрерывного снизу и сверху многозначного отображения многих переменных, если область его определения - компакт, а область значений - вьпуклое замкнутое подмножество метризуемого выпуклого компакта.

Библиограффия: 18 названий.

Введение. Селекторы нескольких переменных необходимы,например, в теории дифференциальных включений [1]-[3]. Однако, даже селекторы двух переменных пока мало изучены (см. [4]-[6]), а селекторы большего числа переменных, по-видимому, до сих пор не исследованы. В работе решается задача существования раздельно непрерьвных селекторов и предложены методы, сводяшие ее к задачам о непрерьвных селекторах.

Статья состоит из двух пунктов. В п. 1 объяснены отличия и связи изучаемой задачи и задачи о непрерьвном селекторе ( точнее о непрерывном селекторе одной переменной). В частности, доказано, что решение исходной задачи может быть получено с помощью решения задачи о непрерывном селекторе специально построенного многозначного отображения. Однако, неметризуемость пространства значений этого многозначного отображения не позволяет дать удовлетворительные решения исходной задачи.

В п. 2 первоначальная задача вновь включена в общую задачу о селекторе (одной переменной), непрерывном на заданной системе множеств. К решению этой задачи привлечена теория сублинейных операторов: методом сублинейных операторов [7] задачи существования искомых селекторов сведены к эквивалентньм задачам субдифференцирования сублинейных операторов.

1. Начнем с ряда определений. Пусть $K$ - вьпукльй компакт отделимого локально вьпуклого пространства $Z$. Через $\operatorname{comp}(K)$ обозначим совокупность всех непустых выпуклых и компактных подмножеств из $K$. В $\operatorname{comp}(K)$ рассмотрим две топологии: топологию Хаусдорфа, в которой базис окрестностей точки $A \in \operatorname{comp}(K)$ состоит из множеств вида

$$
\{B \in \operatorname{comp}(K): A \subset B+V, B \subset A+V\},
$$

где $V$ - базисная окрестность нуля в $Z$, и топологию Вьеториса, назьваемую также экспоненциальной или конечной, определяемую множествами

$$
\{A \in \operatorname{comp}(K): A \cap U \neq \varnothing\}, \quad\{A \in \operatorname{comp}(K): A \subseteq U\}
$$

Работа выполнена при частичной финансовой поддержке Российского фонда фундаментальных исследований, грант № 93-01-00264. 
где $U$ - открытые множества в $K$.

Пусть

$$
T=T_{1} \times T_{2} \times \cdots \times T_{n}
$$

- прямое произведение хаусдорфовых (отделимых) топологических пространств $T_{1}, T_{2}$, $\ldots, T_{n}$, где $n \geqslant 1$. Рассмотрим отображение $\Phi: T \rightarrow \operatorname{comp}(K)$, которое, как обычно, назьвается многозначньм отображением $T$ в $K$, точнее, многозначным отображением $n$ переменных. Если ясно из контекста, то выражение " $n$ переменных" всюду опускается. Это соглашение относится и к другим определениям, в частности, к понятию "селектор". Отображение $\varphi: T \rightarrow K$ назьвается селектором $\Phi$, когда для всех $t \in T$ выполнено включение $\varphi(t) \in \Phi(t)$. Если отображение $\varphi$ непрерывно, то селектор $\varphi$ назьвается непрерывным селектором.

Для $n \geqslant 2$ назовем раздельно непрерывным селектор, которьй непрерывен по каждой переменной в отдельности.

Напомним, что многозначное отображение $\Phi: T \rightarrow \operatorname{comp}(K)$ полунепрерывно снизу (сверху), если для всякого открытого (замкнутого) множества $U$ в $K$ множество $\{t \in T: \Phi(t) \cap U \neq \varnothing\}$ открыто (замкнуто) или, в двойственной формулировке, если для каждого замкнутого (открытого) множества $U$ в $K$ множество $\{t \in T: \Phi(t) \subseteq U\}$ замкнуто (открыто).

Если $n \geqslant 2$, то стандартным образом введем понятие многозначного отображения раздельно полунепрерьвного снизу (сверху), а также раздельно непрерывного в топологии Хаусдорфа или Вьеториса.

ЗАДАчА 1. Для каких пространств $T$, вьпуклых компактов $K \subseteq Z$ и многозначных отображений $\Phi: T \rightarrow \operatorname{comp}(K)$ при любом выборе $t_{0} \in T$ и $k_{0} \in \Phi\left(t_{0}\right)$ существуют раздельно непрерьвные селекторы $\varphi$, удовлетворяющие условию

$$
\varphi\left(t_{0}\right)=k_{0} ?
$$

Иначе говоря, ищется не одиночный селектор, а цельй ансамбль селекторов, целиком заполняющих график многозначного отображения.

В пионерской работе [8] Э. Майкл ввел условие (4) и показал, что для любых $T, K$ выполнение этого условия в задаче о непрерьвном селекторе влечет полунепрерывность снизу многозначного отображения. Повторив рассуждения Э. Майкла в поставленной выше задаче, получим, что для всех $T_{1}, T_{2}, \ldots, T_{n}$ и для всякого выпуклого компакта $K$ необходимьм условием существования раздельно непрерьвных селекторов является раздельная полунепрерывность снизу многозначного отображения. Однако, это условие не будет достаточным в отличие от задачи о непрерьвном селекторе, где необходимое условие является обычно достаточным (например, в [9] доказано, что это так, если $T$ - паракомпакт, а $K$ - вьпукльй метризуемьй компакт). Рассмотрим простой

ПримеР. Пусть $K=[-1,1], T_{2}=[0,1], T_{1}=\mathbb{N}^{*}=\mathbb{N} \cup\{\omega\}$ - одноточечная компактификация Александрова натурального ряда $\mathbb{N}$. Занумеруем все рациональные числа в $T_{2}$ последовательностью $r_{n}$. Определим $\Phi: T_{1} \times T_{2} \rightarrow \operatorname{comp}(K)$ так, что

$$
\Phi\left(t_{1}, t_{2}\right)= \begin{cases}\{0\}, & \text { если } t_{1}=\omega, \\ \left\{\frac{1}{2}\right\}, & \text { если } t_{1}=n, t_{2}=r_{n}, \\ {[-1,1]} & \text { в остальных точках. }\end{cases}
$$

Легко проверить, что $\Phi$ раздельно полунепрерьвно снизу. Покажем, что $\Phi$ не имеет раздельно непрерьвного селектора. Допустим, что такой селектор $\varphi\left(t_{1}, t_{2}\right)$ существует. 
Тогда найдем открытый интервал $I_{1}$, содержащий точку $r_{1}$, такой, что $\varphi\left(1, t_{2}\right) \geqslant 1 / 4$ для $t_{2} \in \bar{I}_{1}$, где черта означает замыкание интервала $I_{1}$. Положим $n_{1}=1$. Далее выберем $n_{2}>n_{1}$, при котором $r_{n_{2}} \in I_{1}$. Теперь укажем открытьй интервал $I_{2}$, содержащий точку $r_{n_{2}}$ и целиком лежащий в $I_{1}$, такой, что для всех $t_{2} \in \bar{I}_{2}$ верно неравенство $\varphi\left(n_{2}, t_{2}\right) \geqslant 1 / 4$. Продолжая этот процесс по индукции, построим возрастающую последовательность натуральных чисел $n_{k}$, последовательность вложенных открытых интервалов $I_{k}$, для которых

$$
\varphi\left(n_{k}, t_{2}\right) \geqslant \frac{1}{4}, \quad t_{2} \in \bar{I}_{2} .
$$

Поэтому существует точка $t_{2}^{0} \in \bigcap_{k=1}^{\infty} \bar{I}_{k}$, в которой неравенство (6) справедливо одновременно для всех номеров $\left\{n_{k}\right\}_{k=1}^{\infty}$. Переходя к пределу в (6) при $k \rightarrow \infty$ и используя непрерьвность селектора по переменной $t_{1}$, получим неравенство $\varphi\left(\omega, t_{2}^{0}\right)>0$. С другой стороны, в силу $(5)$ имеем равенство $\varphi\left(\omega, t_{2}^{0}\right)=0$. Полученное противоречие доказьвает, что $\Phi$ не имеет раздельно непрерьвных селекторов.

ЗАмЕчАниЕ. Отсутствие раздельно непрерывных селекторов и необходимость введения дополнительных условий на многозначное отображение, обеспечиваюших решение задачи 1 , объясняет также следующая конструкция.

Конструкция. Для простоты ограничимся двумя переменными. Общий случай изучается аналогично. Рассмотрим вместе с многозначным отображением $\Phi: X \times Y \rightarrow$ $\operatorname{comp}(K)$, где $X$ и $Y$ - компакты, две серии многозначных отображений

$$
\Phi_{x}: Y \rightarrow \operatorname{comp}(K), \quad \Phi_{y}: X \rightarrow \operatorname{comp}(K),
$$

определенных равенствами $\Phi_{x}(y)=\Phi_{y}(x)=\Phi(x, y)$ для всех $x \in X, y \in Y$. Предположим, что вьпуклый компакт $K$ метризуем, а $\Phi$ раздельно полунепрерьвно снизу. Тогда все многозначные отображения (7), очевидно, полунепрерывны снизу и согласно теореме Майкла о непрерьвньх селекторах (см., например, [9]) имеют непрерьвные селекторы.

Введем следующие многозначные отображения. Через $C_{p}(Y, K)$ обозначим пространство всех непрерывных отображений $Y$ в $K$ с топологией поточечной сходимости. Очевидно, что $C_{p}(Y, K) \subseteq C_{p}(Y, Z)$ и является выпукльм. Пусть $\xi$ есть многозначное отображение $X$ в $C_{p}(Y, K)$, которое каждой точке $x \in X$ сопоставляет множество всех непрерьвных селекторов многозначного отображения $\Phi_{x}$. Аналогично введем многозначное отображение $\eta: Y \rightarrow C_{p}(X, K)$.

Очевидно, что значениями многозначных отображений $\xi$ и $\eta$ являются непустые, выпуклые и замкнутые множества. Полезность введенных отображений $\xi$ и $\eta$ показьвает

ПРЕДЛОЖЕНИЕ 1. Следующие условия әквивалентны:

1) отобрахсение $\Phi$ имеет раздельно непрерывный селектор;

2) отображсение $\xi$ имеет непрерывный селектор;

3) отобрахсение $\eta$ имеет непрерывный селектор.

ДокАЗАТЕЛЬСТВО. В силу симметрии определения $\xi$ и $\eta$ достаточно установить эквивалентность первых двух утверждений. Возьмем раздельно непрерьвньй селектор $\varphi$ многозначного отображения $\Phi$. Тогда отображение $y \mapsto \varphi(x, y)$ будет непрерывным селектором $\Phi_{x}$ при любых $x \in X$. Поэтому отображение $x \mapsto(y \mapsto \varphi(x, y))$ есть искомьй непрерьвньй селектор $\xi$. Обратно, если $\varphi$ - непрерьвньй селектор $\xi$, то отображение $(x, y) \mapsto \varphi(x)(y)$ будет раздельно непрерывным селектором $\Phi$. Предложение 1 доказано.

Следующее предложение показывает, что для отображений $\xi$ и $\eta$ выполнено необходимое условие существования непрерьвных селекторов. 
ПРЕДЛОЖЕНИЕ 2. Пусть $K$ - метризуемый выпуклый компакт, а $\Phi$ раздельно полунепрерывно снизу. Тогда отображения $\xi$ и $\eta$ полунепрерывны снизу.

ДокАЗАТЕЛЬСтво. Так как $\xi$ и $\eta$ равноправны, достаточно проверить полунепрерьвность снизу одного из них, например $\xi$. Рассмотрим поведение отображения $\xi$ в произвольной точке $x_{0} \in X$. Предположим, что селектор $\varphi_{0} \in \xi\left(x_{0}\right)$ выбран произвольно. Пусть $U$ - окрестность нуля в $C_{p}(Y, Z)$ вида $U=U\left(W, y_{0}\right)=\left\{f: f\left(y_{0}\right) \in W\right\}$, где $W-$ окрестность нуля в $Z$, а $y_{0} \in Y$. Для доказательства полунепрерывности снизу отображения $\xi$ достаточно для любой точки $x_{0} \in X$ найти окрестность $V\left(x_{0}\right)$ такую, что при всех $x \in V\left(x_{0}\right)$ имеет место соотношение $\xi(x) \cap\left(\varphi_{0}+U\right) \neq \varnothing$. В качестве $V\left(x_{0}\right)$ возьмем окрестность точки $x_{0}$, в которой $\Phi\left(x, y_{0}\right) \cap\left(\varphi_{0}\left(y_{0}\right)+W\right) \neq \varnothing$ для всех $x \in V\left(x_{0}\right)$. Сушествование такой окрестности следует из полунепрерывности снизу $\Phi$ по переменной $x$ в точке $y_{0} \in Y$. Действительно, окрестность $V\left(x_{0}\right)$ является искомой, потому что для любой точки $x \in V\left(x_{0}\right)$ найдется точка $z_{x} \in K$, которая в силу последнего соотношения принадлежит как $\Phi\left(x, y_{0}\right)$, так и $\varphi_{0}\left(y_{0}\right)+W$. Так как $\Phi(x, y)$ полунепрерьвно снизу по переменной $y$ при фиксированном $x$, существует непрерьвньй селектор $\varphi_{x}$ отображения $\Phi_{x}$ такой, что $\varphi_{x}\left(y_{0}\right)=z_{x}$. Поэтому для любого $x \in V\left(x_{0}\right)$ найдена общая точка $\varphi_{x}$, которая по определению принадлежит $\xi$ и $\varphi_{0}+U$. Таким образом, полунепрерьвность снизу отображения $\xi$ установлена и предложение доказано.

ЗАмЕчАниЕ. Аналогично доказывается, что если в условиях предложения 2 дополнительно $\Phi$ полунепрерьвно сверху, то $\xi, \eta$ также полунепрерьвны сверху.

Итак, задача 1 сведена к задаче о непрерьвном селекторе многозначных отображений $\xi$ и $\eta$. Для них выполнены необходимые условия существования непрерьвных селекторов (предложение 2). Однако, $\xi$ и $\eta$ могут не иметь непрерывных селекторов, потому что, во-первых, пространства их значений неметризуемы и некомпактны, во-вторых, значения $\xi$ и $\eta$ в каждой точке тоже некомпактны. В связи с этим применить теоремы о непрерьвных селекторах с неметризуемыми пространствами значений (см. [10]-[12]) в такой ситуации вряд ли возможно. С другой стороны, можно поставить дополнительную задачу нахождения компактнозначных отображений, вписанных в $\xi$ и $\eta$. Однако, даже при условии решения этой интересной и сложной задачи решение задачи 1 может потребовать весьма жестких условий на компакт $T$, например метризуемости или вполне экстремальной несвязности.

В следующем пункте задача 1 решена для произвольного компакта, но при дополнительном условии: многозначное отображение раздельно полунепрерьвно сверху.

2. В этом пункте изучим исходную задачу, когда

1) $T_{1}, T_{2}, \ldots, T_{n}$ - компакты, следовательно, по теореме Тихонова и $T$ вида $(3)-$ тоже компакт;

2) вьпукльй компакт $K \subseteq Z$ метризуем;

3) многозначное отображение $\Phi: T \rightarrow \operatorname{comp}(K)$ раздельно полунепрерьвно снизу и сверху одновременно.

Справедлива

ТеОрема 1. В условиях 1)-3) всякое многозначное отображсение $\Phi: T \rightarrow$ $\operatorname{comp}(K)$ имеет раздельно непрерывные селекторы, удовлетворяющие условию (4).

ЗАмЕчАниЕ. Теорема 1 является простым следствием более общей теоремы 2 (см. ниже).

Рассмотрим обобщение задачи 1.

Пусть $T$ - произвольный компакт, необязательно вида (3). Зададим на нем произвольную систему $\Omega$ замкнутьх множеств $\omega \in \Omega$. Будем говорить, что отображение, 
заданное на $T$, является $\Omega$-непрерьвным, если его сужение на любое множество $\omega \in \Omega$ непрерьвно. Аналогичное соглашение применяется в дальнейшем и для других свойств отображений, например для полунепрерьвности снизу или сверху многозначных отображений, и т.п. В соответствии с этим селектор, заданный на $T$, назовем $\Omega$-непрерывным.м, если отображение $\varphi$ является $\Omega$-непрерывным.

Сформулируем очевидное обобщение задачи 1.

ЗАДАчА 2. Для каких компактов $T$, вьпуклых компактов $K \subseteq Z$, семейств $\Omega$ и многозначных отображений $\Phi: T \rightarrow \operatorname{comp}(K)$ сушествуют $\Omega$-непрерьвные селекторы, удовлетворяющие условию (4)?

Основным результатом является

ТЕОРема 2. Пусть $T$ - компакт с любой системой $\Omega$ замкнутых множеств, а $K \subseteq Z$ - выпуклый метризуемый компакт. Тогда каждое $\Omega$-полунепрерывное снизу и сверху многозначное отображение $\Phi: T \rightarrow \operatorname{comp}(K)$ имеет $\Omega$-непрерывные селекторы, удовлетворяющие условию (4).

ЛЕммА. Выпуклый метризуемый компакт $K$ в $Z$ аффинно гомеоморфен выпуклому метризуемому компакту $K_{1}$ из сопрязсенного банахова пространства $X^{\prime} c$ топологией $\sigma\left(X^{\prime}, X\right)$, где $X$ - сепарабельное банахово пространство.

ДокАЗАТЕЛЬСтво. Рассмотрим пространство $C(K)$ непрерывных функций, заданных на $K$, с топологией равномерной сходимости. Известно [13], что $C(K)=X$ - сепарабельное банахово пространство. Искомым компактом $K_{1}$ будет множество мер Дирака $\left\{\varepsilon_{k}: k \in K\right\} \subseteq X^{\prime}$.

Согласно этой лемме доказательство теоремы 2 достаточно провести в частном случае, когда $Z=X^{\prime}$ с топологией $\sigma\left(X^{\prime}, X\right)$, где $X$ - сепарабельное банахово пространство.

Метод сублинейных операторов. На вербальном уровне этот метод [7] предполагает наличие класса сублинейных операторов $\mathscr{P}$ и его подкласса $\mathscr{L}$ линейных операторов, класса $\mathfrak{M}$ многозначных отображений с подклассом $\mathfrak{N}$ всех его однозначных отображений и взаимно однозначного отображения между ними. Точнее, взаимно однозначное отображение $\tau: \mathscr{P} \rightarrow \mathfrak{M}$ назовем представлением $\mathscr{P}$, если

1) $\tau(\mathscr{P})=\mathfrak{M}$ и $\tau(\mathscr{L})=\mathfrak{N}$;

2) линейньй оператор $u \in \mathscr{L}$ входит в субдифференциал $\partial Р$ сублинейного оператора $P \in \mathscr{P}$ в том и только том случае, когда $\tau(u)$ является селектором $\tau(P)$.

Классы. Прежде чем ввести классы $\mathscr{P}, \mathscr{L}, \mathfrak{M}$ и $\mathfrak{N}$, напомним определение сублинейного оператора и введем одно полуупорядоченное пространство.

Отображение $P: X \rightarrow Y$, где $X, Y$ - векторные пространства, причем $Y$ полуупорядочено с помошью конуса положительных элементов, называется сублинейным оператором, если оно субаддитивно и положительно однородно, т.е.

$$
P\left(x_{1}+x_{2}\right) \leqslant P x_{1}+P x_{2}, \quad P\left(\lambda x_{1}\right)=\lambda P x_{1}, \quad \lambda \geqslant 0, \quad x_{1}, x_{2} \in X .
$$

В качестве $Y$ рассмотрим пространство $C_{\Omega}(T)$ всех вещественнозначных ограниченных и $\Omega$-непрерьвных функций, заданных на $T$. С обычными операциями сложения и умножения на числа оно превращается в векторное пространство. Наделим его топологией равномерной сходимости на всем $T$ и полуупорядочим естественным образом. Тогда $C_{\Omega}(T)$ является KВ-линеалом ограниченных элементов [14]. 
Класс $\mathscr{P}$ состоит из всех непрерывных сублинейных операторов $P: X \rightarrow C_{\Omega}(T)$, где компакт $T$, система $\Omega$ замкнутых подмножеств $T$ и сепарабельное банахово пространство выбраны произвольно. Подкласс $\mathscr{L}$ класса $\mathscr{P}$ состоит из всех линейных непрерьвных операторов $u: X \rightarrow C_{\Omega}(T)$. Пусть $\mathfrak{M}$ - класс всех $\Omega$-непрерывных в топологии Хаусдорфа многозначных отображений $\Phi: T \rightarrow \operatorname{comp}(K)$, где компакт $T$, выпукльй компакт $K$ в $\left(X^{\prime}, \sigma\left(X^{\prime}, X\right)\right)$ и сепарабельное банахово пространство $X$ выбраныпроизвольно, а $\mathfrak{N}$ подкласс $\mathfrak{M}$, состоящий из однозначных отображений $T$ в $K$.

Для построения отображения $\tau$ удобно ввести еще одно определение, которое позволит нам рассматривать вместо $\Phi: T \rightarrow \operatorname{comp}(K)$ многозначное отображение $T$ в $X^{\prime}$. Скажем, что многозначное отображение $\Phi: T \rightarrow X^{\prime}$ ограничено, если

$$
\sup \left\{\left\|x^{\prime}\right\|: x^{\prime} \in \Phi(t), t \in T\right\} \leqslant \beta<+\infty .
$$

Рассмотрим теперь $\operatorname{conv}\left(X^{\prime}\right)$ - совокупность всех непустых выпуклых компактных в топологии $\sigma\left(X^{\prime}, X\right)$ множеств сопряженного пространства $X^{\prime}$ с топологиями Хаусдорфа и Вьеториса, определяемыми так же, как и для $\operatorname{comp}(K)$ формулами $(1)$ и $(2)$ соответственно. Очевидно, что класс $\mathfrak{M}$ можно определить и как класс $\Omega$-непрерьвных по Хаусдорфу ограниченных отображений $\Phi: T \rightarrow \operatorname{conv}\left(X^{\prime}\right)$.

Представление. Напомним, что линейньй непрерьвньй оператор $u: X \rightarrow Y$ является опорным к непрерьвному сублинейному оператору $P: X \rightarrow Y$, если для всех $x \in X$ справедливо неравенство $u x \leqslant P x$. Совокупность всех непрерьвных операторов опорных к $P$ назьвается его субдифференииалом и обозначается символом $\partial P$. Пусть $X-$ произвольное банахово пространство и $P: X \rightarrow C_{\Omega}(T)$ - любой непрерывный сублинейный оператор. Каждому $P$ сопоставим многозначное отображение $\Phi_{P}$, определенное формулой

$$
t \mapsto \partial(x \mapsto P x(t)), \quad x \in X
$$

и действующее из $T$ в $\operatorname{conv}\left(X^{\prime}\right)$.

Отображение $P$ в $\Phi_{P}$ будем рассматривать как представление $\tau$. Справедлива

Теорема 3. Для любого банахова пространства $X$ отображсение $\Phi_{P}: T \rightarrow$ $\operatorname{conv}\left(X^{\prime}\right)$ является $\Omega$-непрерывным в топологии Хаусдорфа и ограниченным. Обратно, каждое $\Omega$-непрерывное в топологии Хаусдорфа и ограниченное отображсение $\Phi: T \rightarrow \operatorname{conv}\left(X^{\prime}\right)$ порождает формулой

$$
P x(t)=\sup \left\{\left\langle x, x^{\prime}\right\rangle: x^{\prime} \in \Phi(t)\right\}, \quad t \in T, \quad x \in X,
$$

непрерывный сублинейный оператор $P: X \rightarrow C_{\Omega}(T)$, для которого $\Phi_{P}=\Phi$.

ДокАЗАТЕЛЬСтво. Теорема 3 в частном случае, когда $\Omega$ состоит из одного множества $T$, получена в [15] (доказательство см. в [16]). Общий случай сводится к частному, если вместе с $P: X \rightarrow C_{\Omega}(T)$ рассмотреть семейство непрерьвных сублинейных операторов $P_{\omega}: X \rightarrow C(\omega)$, заданных как "сужение" оператора $P$, т.е. $P_{\omega} x=\left.P x\right|_{\omega}, x \in X$, и учесть равенство $\tau\left(P_{\omega}\right)=\left.\Phi_{P}\right|_{\omega}, \omega \in \Omega($ см. формулы $(9)$ и $(10))$. Напомним, что условие ограниченности, когда $\Omega=\{T\}$, следует из непрерывности $\Phi_{P}$ в топологии Хаусдорфа, причем константу $\beta$ в (8) можно считать равной $\|P\|$ (см. [14], [15]). Далее ясно, что непрерьвность сублинейного оператора $P: X \rightarrow C_{\Omega}(T)$ эквивалентна его ограниченности: $\|P\|<\infty$. Поэтому для $\Phi_{P}$ в качестве константы $\beta$ вновь можно взять $\|P\|$. Однако, если некоторое многозначное отображение $\Phi: T \rightarrow \operatorname{conv}\left(X^{\prime}\right)$ ограничено на каждом компакте $\omega \in \Omega$, то оно вовсе необязательно является ограниченным на всем $T$. Следовательно, в теореме 3 вместе с условием $\Omega$-непрерывности необходимо и условие ограниченности $\Phi$. Теорема доказана. 
СлЕдСтвИЕ. Линейные непрерывные операторы $u: X \rightarrow C_{\Omega}(T)$, и только они, имеют в качестве представлений однозначные и ограниченные отображения $T$ в $X^{\prime}$.

Следующая простая, но принципиально важная, теорема устанавливает связь между субдифференцируемостью и изучаемой задачей о селекторе. Напомним, что сублинейньй оператор называется субдифферениируемым, если его субдифференциал есть непустое множество, и несубдифферениируемым - в противном случае.

ТеОрема 4. Непрерывный сублинейный оператор $P: X \rightarrow C_{\Omega}(T)$ субдифферениируем тогда и только тогда, когда его представление $\tau(P)$ имеет $\Omega$-непрерывный селектор.

Доказательство теоремы следует из формул (9), (10) и следствия для любых банаховых пространств.

Перейдем к доказательству теоремы 2, считая теперь во всех предыдущих рассуждениях $X$ сепарабельным банаховьм пространством.

ДОКАЗАТЕЛЬСТво ТЕОРЕмЫ 2. Рассмотрим $\Omega$-полунепрерывное снизу и сверху многозначное отображение $\Phi: T \rightarrow \operatorname{comp}(K)$. Используя формулу $(2)$, заключаем, что $\Phi$ является $\Omega$-непрерьвньм в топологии Вьеториса. Учитьвая совпадение топологий Хаусдорфа и Вьеториса на $\operatorname{comp}(K)[17$, с. 208,254$]$, устанавливаем $\Omega$-непрерьвность $\Phi$ в топологии Хаусдорфа. Согласно лемме можно считать, что вьпуклый компакт $K$ содержится в $Z=X^{\prime}$, где $X$ - сепарабельное банахово пространство со слабой топологией $\sigma\left(X^{\prime}, X\right)$. Так как компакт $K$ в $X^{\prime}$ ограничен, можно считать, что $\Phi$ является ограниченньм отображением $T$ в $\operatorname{conv}\left(X^{\prime}\right)$. Следовательно, можно применить теорему 3 и представить $\Phi$ как $\Phi=\Phi_{P}=\tau(P)$, где непрерьвный сублинейньй оператор $P: X \rightarrow C_{\Omega}(T)$ вычисляется по формуле (10). Как уже отмечалось, $C_{\Omega}(T)$ является КВ-линеалом ограниченных элементов. Тем самым, $C_{\Omega}(T)$ - пространство Линденштраусса, и оператор $P$ субдифференцируем [18]. Подчеркнем, что только в этом месте использована по существу сепарабельность банахова пространства $X$. Пусть $u \in \partial P$. В силу теоремы $4 \tau(u)=\varphi$ является $\Omega$-непрерьвньм селектором многозначного отображения $\Phi$. Необходимо добавить, что этот селектор согласно (9) имеет вид

$$
\varphi: t \mapsto(x \mapsto u x(t)), \quad x \in X, \quad t \in T .
$$

Иными словами, $u$ и $\varphi$ связаны соотношением

$$
u x(t)=\langle x, \varphi(t)\rangle, \quad x \in X, \quad t \in T .
$$

Осталось доказать, что найдется $\Omega$-непрерьвньй селектор, удовлетворяющий условию (4), т.е. для любых точек $t_{0} \in T$ и $x_{0}^{\prime} \in K$ требуется указать $\Omega$-непрерывный селектор, для которого верно равенство $\varphi\left(t_{0}\right)=x_{0}^{\prime}$. Для этого возьмем субдифференциал $\partial P$ фигурируюшего вьше непрерывного сублинейного оператора $P$ и отметим, что кроме субдифференцируемости оператора $P$ доказана формула

$$
\sup \{u x(t): u \in \partial P\}=P x(t), \quad x \in X, \quad t \in T
$$

(ср. с формулой (2) в [18]). Рассмотрим также множество $S$ всех $\Omega$-непрерывных селекторов многозначного отображения $\Phi$. Между $u \in \partial P$ и $\varphi \in S$ есть взаимно однозначное соответствие, определяемое соотношениями (11) и (12). Поэтому (13) допускает еще одно выражение:

$$
\sup \{\langle x, \varphi(t)\rangle: \varphi \in S\}=P x(t), \quad x \in X, \quad t \in T .
$$


Для завершения доказательства заметим, что множество селекторов $S$ замкнуто в топологии поточечной сходимости пространства $\Omega$-непрерывных ограниченных отображений $T$ в $X^{\prime}$ и в каждой точке $t \in T$ множества $S(t) \subseteq X^{\prime}$ выпуклы. Таким образом, $S\left(t_{0}\right)$ - вьпуклый компакт в $\operatorname{conv}\left(X^{\prime}\right)$, совпадаюший согласно двойственности Минковского и (14) с $\partial\left(x \mapsto P x\left(t_{0}\right)\right), x \in X$. Стало быть, обязательно найдется $\varphi \in S$ такой, что $\varphi\left(t_{0}\right)=x_{0}^{\prime}$. Теорема доказана.

Заключительные замечания. Предлагаемые методы можно использовать и при решении задач о $\Omega$-непрерывном селекторе для неметризуемых выпуклых компактов $K$. Например, используя [12], теорему 2 можно доказать в предположении метризуемости компакта $T$, счетности семейства $\Omega$, если $K$ - вьпукльй компакт Эберлейна.

Эти методы эффективны, если $T$ не является компактным. Только в этом случае надо предварительно доказать соответствующие теоремы о представлении сублинейных операторов со значениями в пространстве $C_{\Omega}(T)$.

Не вызывает принципиальных затруднений перенесение этих методов на случай, когда рассматриваются селекторы многих переменных, непрерывные по одним и измеримые или борелевские по другим переменньм. Однако, нужны новые теоремы о представлении сублинейных операторов измеримыми и борелевскими отображениями.

\section{СПИСОК ЦИТИРОВАННОЙ ЛИТЕРАТУРЫ}

[1] Aubin I. P., Cellina A. Differential Inclusions. Berlin: Springer, 1984.

[2] Толстоногов А. А. Дифференциальные включения в банаховом пространстве. Новосибирск: Наука, 1986.

[3] Bressan A., Colombo G. Extensions and selections of maps with decomposable values // Studia Math. 1988. V. 90. №1. P. 69-86.

[4] Kucia A., Nowak A. On Caratheodory type selectors in a Hilbert space // Ann. Math. Sil. 1986. № 14. P. 47-52.

[5] Phan V.C. A density theorem with an application in relaxation of nonconvex-valued differential equations // J. Math. Anal. Appl. 1987. V. 124. №1. P. 1-14.

[6] Slezak W. Concerning continuous selectors for multifunctions defined on product spaces // Problemy Mat. 1987. № 8. P. 31-46.

[7] Линке Ю.Э. Метод сублинейных операторов и задачи о селекторах // Докл. РАН. 1996. T. 347. № 4. C. 446-448.

[8] Michael E. Continuous selections. 1 // Ann. Math. 1956. V. 63. № 2. P. 361-382.

[9] Michael E. A selection theorem // Proc. Amer. Math. Soc. 1966. V. 17. P. 1404-1407.

[10] Corson H. H., Lindenstraus I. Continuous selections with non-metrizable range // Trans. Amer. Math. Soc. 1966. V. 121. № 2. P. 492-504.

[11] Hasumi M. A continuous selection theorem for extremally disconnected spaces // Math. Ann. 1969. V. 179. №2. P. 83-89.

[12] Линке Ю.Э. Сублинейные пространства со значениями в пространствах непрерывных функций // Докл. АН СССР. 1976. Т. 228. № 3. С. 540-542.

[13] Данфорд Н., Шварц Дж. Т. Линейные операторы. Общая теория. М.: ИЛ, 1962.

[14] Вулих Б.З. Введение в теорию полуупорядоченных пространств. М.: Физматгиз, 1961.

[15] Линке Ю. Э. Об опорных множествах сублинейных операторов // Докл. АН СССР. 1972. T. 207. №3. C. 531-533.

[16] Линке Ю. Э. Об опорных множествах сублинейных операторов со значениями в $C(Q) / /$ Оптимизация. Вьп. 8(25). Новосибирск, 1972. С. 52-70.

[17] Бурбаки Н. Общая топология. Основные структуры. М.: Наука, 1968.

[18] Линке Ю.Э. Сублинейные операторы и пространства Линденштраусса // Докл. АН CCCP. 1977. T. 234. № 1. C. 26-29. 